\title{
THE 31ST INTERNATIONAL ASTIN COLLOQUIUM
}

\author{
Porto Cervo, Italy, 19-21 September 2000
}

The 31st International ASTIN Colloquium will be held in Porto Cervo, Italy from the 18th to the 21 st of September 2000.

The Institute of Actuaries of Japan has just passed on the ASTIN banner to the Istituto Italiano degli Attuari whose members feel highly honoured and at the same time feel the responsibility to be entrusted of the organisation of Colloquium ASTIN 2000.

The topics that will be discussed during the Colloquium, cover subjects that we think of great current interest:

1) Alternative models to the probability of ruin in risk theory,

2) Modelling Catastrophic Risks,

3) Technical Management of Health Insurance,

4) Others.

However, we would like to express the wish to receive papers dealing with the new frontiers of the research in Actuarial Science in Non Life!

The Call for Papers sent in May requested submissions by 1st March 2000. Authors are kindly invited to respect this deadline to allow the participants to receive the Proceedings in time for the Colloquium. The Scientific programme of the Colloquium includes also two invited lectures and a round table on Estimating Loss Reserves using Various Methods and Models: a Comparative Analysis.

It will be completed by a particularly significant moment: the prize for the best contribution on analytical expressions suitable to approximate practical claim size distributions, will be awarded at the ASTIN 2000 Meeting.

The working sessions will be held at the Porto Cervo Hotel Conference Centre. Not all the participants will stay at the same hotel at least on the basis of their preference expressed in the pre-registration forms. But in any case, all participants will enjoy the exclusive Costa Smeralda and the scenario of a breathtaking panorama.

We would like you all to appreciate the beauty of this island as well as its culture and traditions. With this aim the Organizing Committee is preparing the Social Programme.

We look forward to meeting any ASTIN colleagues in Porto Cervo and our wish is to receive many good papers to discuss all together.

Arrivederci in Italia

Il Presidente

Prof. Carla Angela Mormino
Further information:

ASTIN 2000 Colloquium Secretariat c/o Iniziative

via Domenico Chelini, 4-00197 Rome, Italy 